M. BIHANI, T. N. ANSARI, L. FINCK, P. P. BORA, J. B. JASINSKI, B. PAVULURI,

D. K. LEAHY*, S. HANDA* (TAKEDA PHARMACEUTICALS INTERNATIONAL, CAMBRIDGE AND UNIVERSITY OF LOUISVILLE, USA)

Scalable $\alpha$-Arylation of Nitriles in Aqueous Micelles Using Ultrasmall Pd Nanoparticles: Surprising Formation of Carbanions in Water

ACS Catal. 2020, 10, 6816-6821.

\section{$\alpha$-Arylation of Nitriles Catalyzed by Palladium Nanoparticles in Proline-Based Micelles}

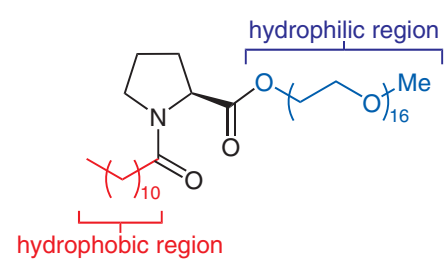

$\underset{\substack{\mathrm{H}_{2} \mathrm{O} \\ \text { micelle formation }}}{\stackrel{\mathrm{K}_{3} \mathrm{PO}_{4} \text { (2.0 equiv) }}{\longrightarrow}}$

proline-based amphiphile
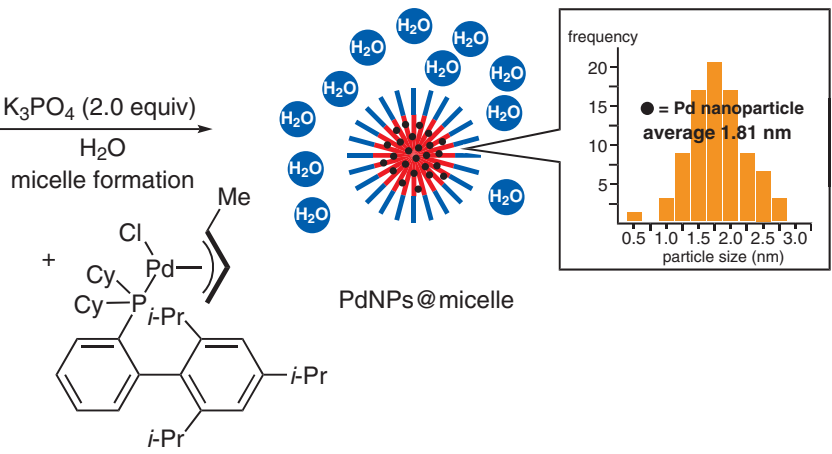

(1)

PdNPs@micelle

\section{Category}

Polymer-Supported

Synthesis

Key words

palladium catalysis

micellar catalysis

nitriles

arylation

aqueous media

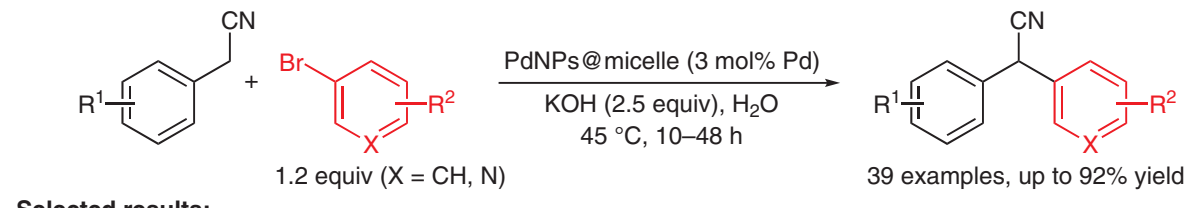<smiles>COc1ccc(C(C#N)c2cccc(OC)c2)cc1</smiles><smiles>COc1ccc(C(C#N)c2ccc(Cl)cc2)cn1</smiles><smiles>COc1ccc(C(C#N)c2cccc3ccccc23)cc1</smiles><smiles>CCc1ccc(C(C#N)c2ccccc2)cc1</smiles>

$\mathrm{R}^{2}=$ OMe $91 \%$ yield $\mathrm{R}^{2}=\mathrm{Cl} \quad 77 \%$ yield $\mathrm{R}^{2}=\mathrm{CF}_{3} 66 \%$ yield<smiles>N#CC(c1ccc(Cl)cc1)c1c2ccccc2cc2ccccc12</smiles>

$85 \%$ yield<smiles></smiles>

$41 \%$ yield<smiles>N#CC(c1ccccc1)c1ccc(NC(=O)OCc2ccccc2)cc1</smiles>

$65 \%$ yield

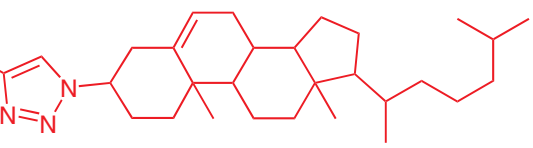

Significance: Palladium nanoparticles in micelles of a proline-based amphiphile (PdNPs@micelles), generated in situ (eq. 1), promoted the $\alpha$-arylation of arylacetonitriles with aryl bromides to give the corresponding $\alpha$-arylated products in up to $92 \%$ yield (eq. 2).
Comment: The PdNPs were characterized by means of ${ }^{31} \mathrm{P}$ NMR, HRTEM, and EDAX analyses. A heterogeneous and amphiphilic vesicular Pd catalyst has been previously reported (see, for example: G. Hamasaka, T. Muto, Y. Uozumi Angew. Chem. Int. Ed. 2011, 50, 4876). 